\title{
Espectáculos y realitys shows y la vulneración al derecho de educación, Chachapoyas, Perú, 2019
}

\section{Shows and Realitys shows and the violation of the right to education, Chachapoyas, Peru, 2019}

\author{
Diego Alexander Segura Guerra ${ }^{1}$, Segundo Roberto Guevara Aranda²
}

\section{RESUMEN}

Esta investigación tuvo por objetivo, determinar la grave vulneración del derecho a la educación por parte de los espectáculos y reality shows, en televisión de señal abierta. El diseño de investigación fue descriptivo no experimental, la población fue 207 estudiantes del primer grado de secundaria de la Institución Educativa Emblemática San Juan de la Libertad, Chachapoyas, la muestra estuvo constituida por 82 estudiantes, el muestro fue aleatorio simple. El instrumento utilizado fue la encuesta. Se encontró que el $84.1 \%$ de los estudiantes observa programas de espectáculos o entretenimiento, el $47.6 \%$ los observa de forma inter diaria, el $72 \%$ lo hace por gusto o agrado; un $84.1 \%$ no se identifica con algún personaje, el $56.1 \%$ indico que el contenido es negativo, el 80.6\% considera que los programas de espectáculos y/o reality shows no contribuyeron a su formación académica. En conclusión, se determinó una grave vulneración al derecho a la educación, al constatarse que la emisión de espectáculos y reality shows, infringe el deber colaboración con el Estado en la educación y en formación moral y cultural; además de transgredir el horario de protección al menor establecido en la Ley 28278.

Palabras claves: sívulneración, educación, deber de colaboración.

\begin{abstract}
The objective of this investigation was to determine the serious violation of the right to education by shows and reality shows, on open signal television. The research design was descriptive, not experimental, the population was 207 first-grade high school students from the San Juan de la Libertad Emblematic Educational Institution, Chachapoyas, the sample consisted of 82 students, the sample was simple random. The instrument used was the survey. It was found that $84.1 \%$ of the students observe shows or entertainment programs, $47.6 \%$ observe them on an inter-daily basis, $72 \%$ do it for pleasure or pleasure; $84.1 \%$ do not identify with a character, $56.1 \%$ indicate that the content is negative, $80.6 \%$ consider that show programs and / or reality shows did not contribute to their academic training. In conclusion, a serious violation of the right to education was determined, when it was found that the broadcasting of shows and reality shows violates the duty of collaboration with the State in education and in moral and cultural training; in addition to violating the child protection schedule established in Law 28278.
\end{abstract}

Keywords: violation, education, duty of collaboration.

${ }^{1}$ Bachiller en Derecho egresado de la Facultad de Derecho y Ciencias Políticas de la Universidad Nacional Toribio Rodríguez de Mendoza 2 Abogado, Magister en Derecho Constitucional, docente de la Facultad de Derecho y Ciencias Políticas, Universidad Nacional Toribio Rodríguez de Mendoza. Correo electrónico: segundo.guevara@untrm.edu.pe 


\section{INTRODUCCIÓN}

En un escenario distópico, en el que el sistema estatal peruano se ha visto orientado por la hegemonía internacional, al desacoplarse de manera sutil y perniciosa de los parámetros imperativos establecidos en el marco constitucional, que desde un punto de vista ideal propicia un Estado de derecho, dando paso a los medios de comunicación, los que envuelven una millonaria empresa en su quehacer diario al emitir y difundir los llamados reality Shows y programas de espectáculos los que, sin lugar a duda, vulneran uno de los derechos esenciales para la vida de todo ciudadano: la educación.

En esa línea de ideas, el Tribunal Constitucional, hace énfasis en que "la educación es un derecho fundamental, intrínseco y un medio indispensable para la plena realización de otros derechos fundamentales, y permite al ciudadano participar plenamente en la vida social y política en sus comunidades." (Expediente $\mathrm{N}^{\circ}$ 0091-2005-AA. Determinación del contenido del derecho a la educación, Fundamento Jurídico $\mathrm{N}^{\circ}$ 06, Tribunal Constitucional).

Haciendo alusión a ello Tomaševski (2000) menciona que "al igual que Jano, el dios de las puertas de la mitología romana, la educación tiene dos caras: la que mira hacia adelante da paso a otros derechos cuando el derecho a la educación está garantizado, en tanto que la mira hacia atrás deja ver que la negativa del derecho a la educación entraña el rechazo de otros derechos"

Si bien es cierto, hoy en día, la televisión juega un rol importante en la educación, ya que los medios de comunicación tienen el deber de colaborar con el Estado en la educación y en la formación moral y cultural, es decir, que coadyuve a la formación del pensamiento crítico y que conlleve a la reflexión analítica.

En respaldo a ello la Ley $N^{\circ} 28278$, Ley de Radio y Televisión, titulo preliminar Artículo II.- Principios para la prestación de los servicios de radiodifusión, establece que "La protección y formación integral de los niños y adolescentes, así como el respeto de la institución familiar"; al mismo tiempo que son intereses prioritarios del Estado, en ese sentido la transmisión de su programación debe estar orientada al desarrollo de las capacidades críticas y analíticas, todo con miras a propiciar una sociedad en la que sus miembros puedan ser consientes de las problemáticas existentes y puedan, brindar soluciones.

Dicho ello, el Tribunal Constitucional establece precedentes respecto al principio de contribución, el cual hace referencia al deber de los medios de comunicación de colaborar de forma solidaria con la formación moral, cívica y cultural de la población, concordante con el principio de coherencia, el cual plantea como necesidad que las distintas maneras y contenidos derivados del proceso educativo mantengan una cohesión, en la cual debe proteger especialmente al niño y al adolescente. (Expediente $\mathrm{N}^{\circ}$ 04232-2004-AA. Principios que rigen el proceso educativo, Fundamento Jurídico $\mathrm{N}^{\circ} 12$, Tribunal Constitucional); ello se vincula de manera directa con el deber de educar con el que cuentan los medios de comunicación, según lo regulado en el Artículo $\mathrm{N}^{\circ} 14$ de la Constitución Política del Perú y su evidente vulneración por parte de los reality show y programas de espectáculos, al apartarse de la finalidad del Estado, de brindar protección al niño y adolescente.

Situación que desde un punto de vista concreto, partiendo de la realidad existente, se evidencia cada vez más en los programas de espectáculos y reality shows la ausencia de mínimos indicios de cualquier tipo de aporte critico o analítico a sus televidentes, en especial a los adolescentes y pre adolescentes que es el sector de la población que más consume este tipo de programas y que son más susceptible de vulnerar su derecho a la educación, pero sobre todo a la educación de calidad; más aún, si es que este tipo de programas que son carentes de cualquier bagaje educacional o cultural, se transmiten en horarios que la Ley $N^{\circ} 28278$ - Ley de Radio y Televisión ha establecido como horario familiar, cual es considerado como horario de protección al menor, el mismo que va desde las 06:00 de la mañana hasta las 10:00 de la noche, horario en el cual según lo estipulado por la misma norma se debe evitar los programas de contenido obsceno o de otra índole; situación que a todas luces se evidencia su inobservancia al emitirse reality show y programas de espectáculos a toda hora del día.

La presente investigación tuvo como Objetivo Determinar el grado de vulneración al derecho a la educación por parte de los espectáculos y reality shows, en la televisión de señal abierta, a los estudiantes del $1^{\text {a }}$ de secundaria de la I.E.E. San Juan de la libertad, Chachapoyas, 2019.

Para ello, se tuvo que cumplir con Objetivos Específicos tales como: Determinar los tipos de entretenimiento que más seducen a observar a los estudiantes; demostrar la influencia negativa de los espectáculos y reality shows; examinar en qué medida los espectáculos y reality shows vulneran el horario de protección al menor establecido en el artículo 40 de la ley 28278 , ley de radio y televisión; establecer el grado de ponderación del derecho a la 
educación respecto a la libertad de empresa, libertad de expresión y de opinión.

\section{MATERIAL Y MÉTODO}

\subsection{Diseño de investigación}

El diseño de investigación fue descriptivo no experimental, de tipo transversal.

\subsection{Población y muestra}

\section{a) Población}

La población estuvo conformada por 207 estudiantes del primer grado de secundaria, distribuidos en las secciones "A", "B", "C", "D", "E", "F", "G", de la I.E.E. "San Juan de la Libertad", Chachapoyas, en el año 2019.

\section{b) Muestra}

Estuvo conformada por 82 estudiantes, seleccionados a través del muestreo aleatorio simple.

\subsection{Métodos}

La técnica utilizada fue la encuesta conteniendo 12 preguntas dirigidas a la muestra de estudiantes del $1^{\circ}$ de secundaria, distribuidos en siete secciones, desde la "A" hasta la "G", a fin de evaluar la vulneración del derecho a la educación.

El instrumento aplicado, objetivamente recayó en la ficha cuestionario, definido como: "El cuestionario es un formulario impreso que los individuos responden por sí mismos sirviendo de instrumento para obtener información deseada, sobre todo a escala masiva. El mismo está compuesto por preguntas previamente elaboradas que son significativas para la investigación y se aplica al universo o muestra de las unidades de análisis" (Velázquez y Rey, 2007).

\section{RESULTADOS}

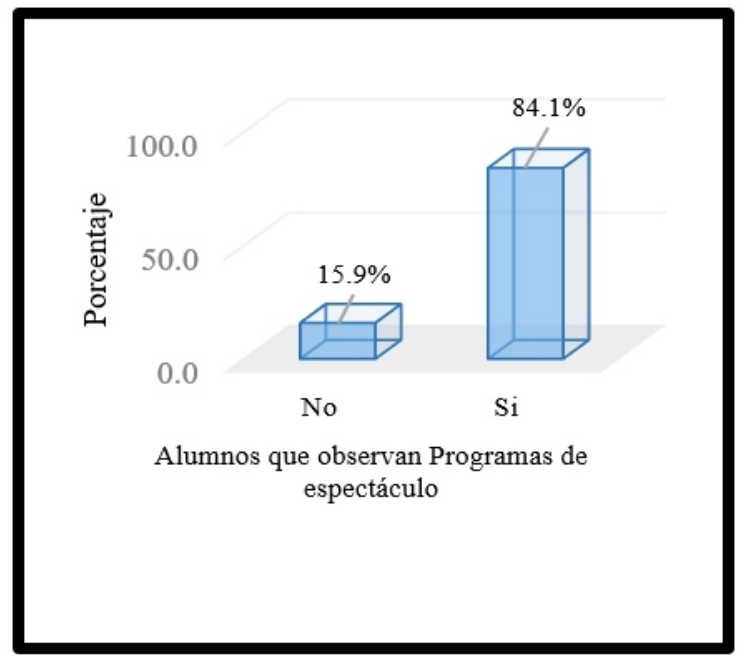

Figura 1. Porcentajes de estudiantes que observaron programas de espectáculo o entretenimiento.

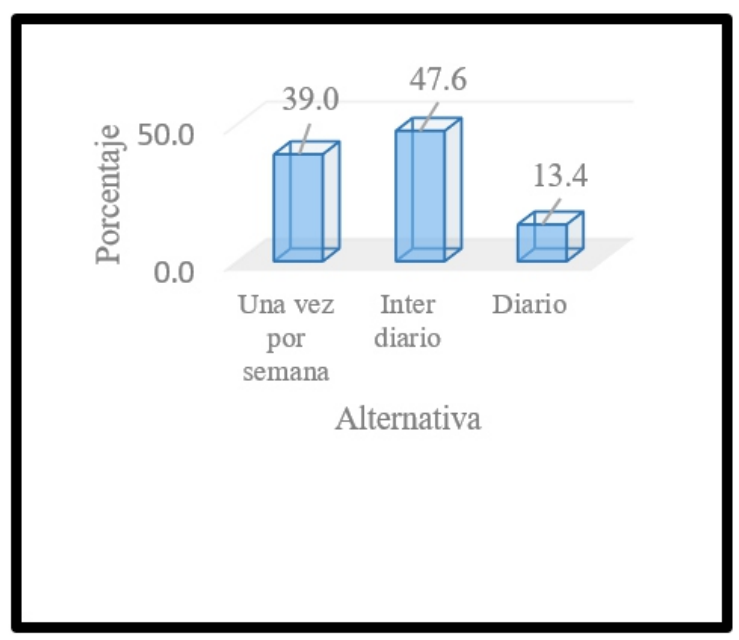

Figura 2. Porcentajes de la regularidad con la que los estudiantes observan programas de espectáculos.

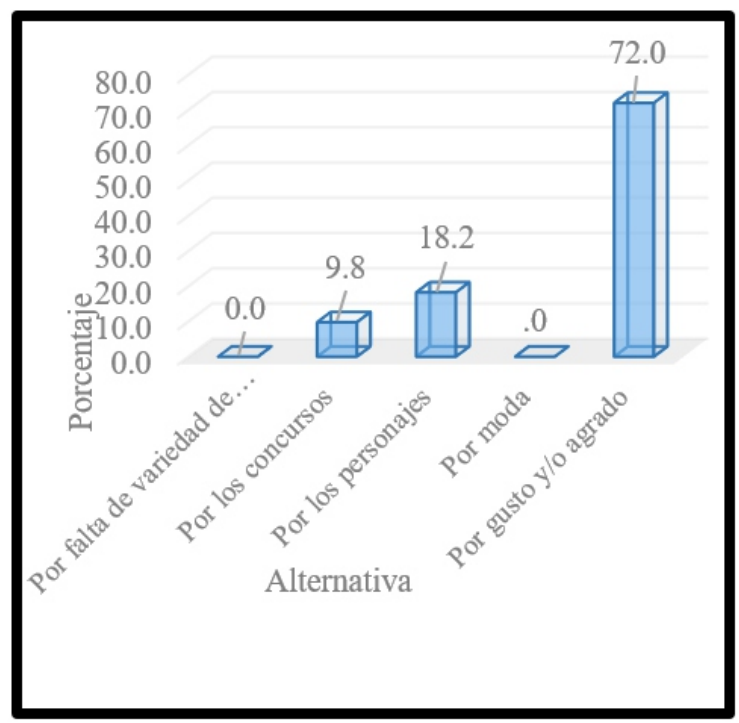

Figura 3. Porcentajes del impulso por observar programas de espectáculos.

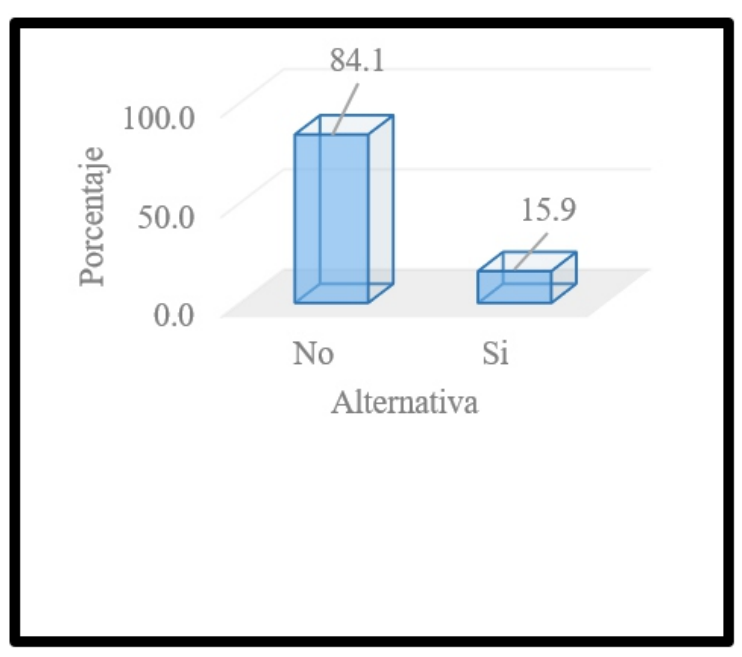


Figura 4. Porcentajes de la identificación con los personajes de los programas de espectáculos y/o reality shows.

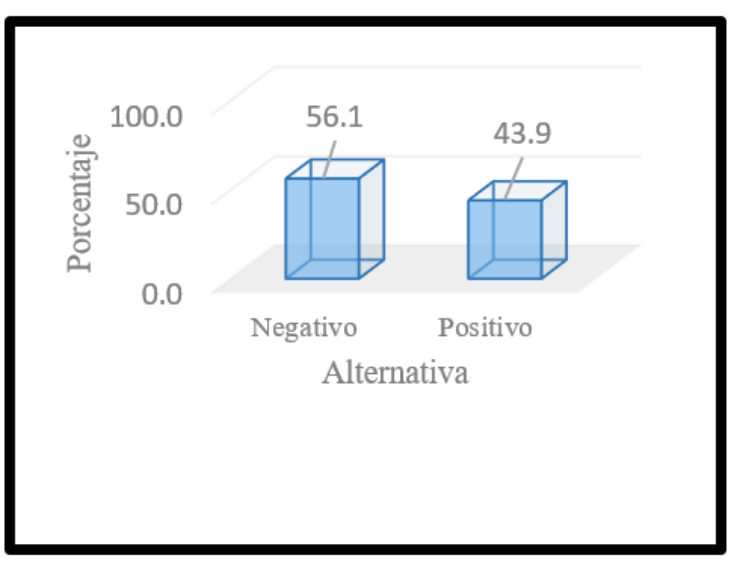

Figura 5: Porcentajes sobre la valoración del contenido de los programas de espectáculos y/o reality shows.

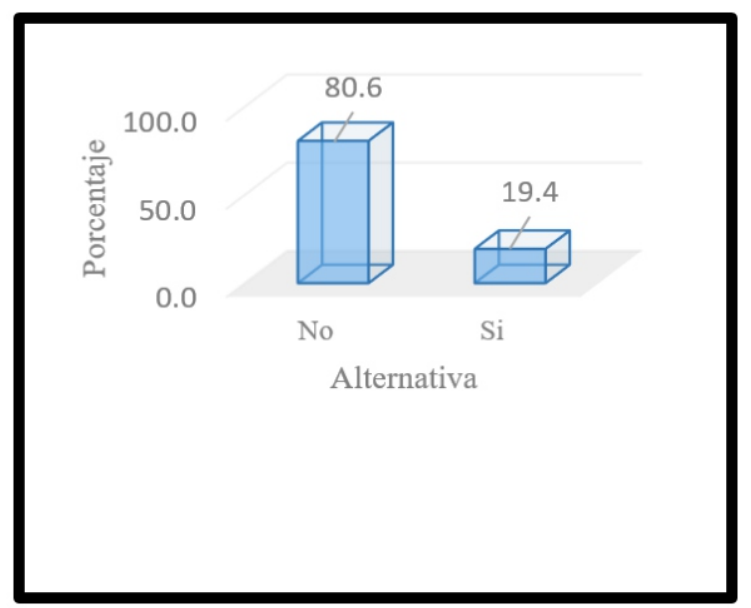

Figura 6. Porcentajes sobre la observación de programas de espectáculo y/o reality shows y la contribución a la formación académica de los estudiantes.

\section{DISCUSIÓN}

Del análisis de la figura 1, según la investigación realizada se ha obtenido que del $100 \%$ de los estudiantes encuestados, el $84,1 \%$ observa programas de espectáculos o entretenimiento ante un $15.9 \%$ que menciona que no lo hace; resultados que coinciden con la investigación realizada por Villón (2016), quien luego del análisis y discusión realizados, arrojó que los encuestados prefieren programas de entretenimiento como los reality shows, sin importar tanto la notabilidad de los personajes que participan; que evitarían realizar casting para participar en estos programas; además, creen que la televisión que se brinda en el Ecuador debe ofertar otro tipo de programación en los horarios familiares.

Del análisis de la figura 2, según la investigación realizada se ha obtenido que del $100 \%$ de los estudiantes encuestados, el 39\% observan programas de espectáculos una vez por semana, un $47.6 \%$ lo hacen inter diario y un $13.4 \%$ diario, resultados que guardan relación con la investigación realizada por el órgano autónomo, plural y consultivo adscrito al Ministerio de Transportes y Comunicaciones Concortv, el que indica: los 'reality' de TV son los programas más vistos por los niños y adolescentes; el estudio revela preocupante realidad. Más del 50\% de menores admiran a los participantes de Esto es Guerra y Combate y quieren ser como ellos, señala Concortv. Estos programas son vistos en familia, aunque ellos siempre deciden qué quieren ver. Los programas de televisión donde participan ellos son los más consumidos por los pequeños de casa. Hoy, los 'chicos reality' se han convertido en los preferidos de los niños y adolescentes del país, al punto de que el $52,8 \%$ de 9 mil 149 menores encuestados, admira a estos personajes y desea ser como uno de ellos.

Así lo revela el estudio sobre consumo televisivo y radial en niños, niñas y adolescentes, del Consejo Consultivo de Radio y Televisión (Concortv), elaborado por Calandria entre el 3 y 27 de julio último. En este se consideró a menores entre 7 y 16 años de 18 ciudades del Perú. Pero, ¿qué es lo que nos enseñan estos programas? ('realities' como Esto es Guerra, Combate, entre otros). "Nos dicen que las chicas debemos vestirnos con ropa muy corta y que debemos tener un buen cuerpo para ser aceptadas", critica Daniela Meza, una menor representante del Consejo Consultivo de Niños, Niñas y Adolescentes de Lima Metropolitana (Chinchay, 2016).

Del análisis de la figura 3, según la investigación realizada se ha obtenido que del 100\% de los estudiantes encuestados, el $9.8 \%$ observa programas de espectáculos por los concursos, mientras que el $18.2 \%$ por los personajes y un $72 \%$ por gusto $y / 0$ agrado. Comparando los resultados obtenidos en la presente investigación con los resultados obtenidos en la investigación de Centeno (2016), encontramos similitud en cuanto considera, que estos programas son producto de la tendencia actual en televisión, no solo nacional, de despertar en los televidentes el morbo, la curiosidad, el interés banal por las ocurrencias en pantalla y los camerinos. De eso se trata el reality, de exponer las supuestas "vivencias reales" de los participantes de este tipo de programas para satisfacer a la teleplatea. Para ello se escogen personajes adecuados (de un determinado perfil) y se preparan guiones para el desarrollo de sus 
actuaciones. Todo es parte de un entramado televisivo bien montado que llega de contrabando a su público objetivo: niños y adolescentes que cada día están a las expectativas de las peleas, romances y escándalos de los participantes.

Del análisis de la figura 4, según la investigación realizada se ha obtenido que del $100 \%$ de los estudiantes encuestados un $84.1 \%$ no se identificaron con los personajes de los programas de espectáculos y realitys show, frente a un $15.9 \%$ que si lo hicieron; en esa línea de ideas coincidimos con Pintado (2015), porque con su investigación logró determinar que los alumnos del décimo de básica del colegio San José La Salle son influenciados por los programas que ven en la televisión, sobre todo reciben una influencia negativa de aquellos programas con contenidos discriminatorios $y$ violentos. Dicha influencia se refleja en el comportamiento de estos jóvenes en sus aulas de clases, en sus hogares y cuando se reúnen con sus amigos; por su puesto, peor es la influencia nociva en adolescentes mucho más tiernos como los de la muestra utilizada.

Del análisis de la figura 5, según la investigación realizada se ha obtenido que del $100 \%$ de los estudiantes encuestados un $56.1 \%$ tiene una apreciación negativa de los programas de espectáculos y/o reality shows, frente a un $43.9 \%$ que tienen una apreciación negativa; resultados que coincide con la problemática desarrollada en el capítulo II del Proyecto Educativo Regional de Amazonas 2007-2021, referido al panorama educativo en la región Amazonas, como parte de las brechas y limitaciones, específicamente cuando se describe sobre el desarraigo, violencia familiar y escolar: "Los medios de comunicación fomentan conductas violentas, libertinaje sexual, información alienante y promueven actitudes consumistas a través de programas, publicidad y modas importadas que debilitan la autoestima, identidad, relaciones de afecto y de comunicación familiar" (Gobierno Regional de Amazonas, 2007).

En esa línea de ideas Galvez (2015) concibió que la libertad de expresión justifica la erradicación de la televisión basura transmitida por señal abierta en el horario familiar cuando se interpreta en razón de sus límites y restricciones con el fin de proteger y salvaguardar la moral y el orden público, además el derecho de libertad de expresión tiene como fundamento político la democracia de lo que se desprende la participación ciudadana, igualdad y el estado constitucional de derecho.

Ante ello cabe destacar, que mediante la difusión de programas de espectáculos y realitys shows de manera deliberada sin la existencia de una regulación adecuada, el estado peruano pondera la libertad de empresa, libertad de expresión y libertad de opinión, frente al derecho a la educación, en vista a la existencia proliferada de este tipo de programas, los cuales según la investigación realizada vulnera límites constitucionales y el horario de protección al menor.

Argumento que se sustenta en el test de ponderación de Robert Alexy (2009), en el sentido que, la ley de ponderación muestra que ésta puede descomponerse en tres pasos. En el primero debe constatarse el grado de incumplimiento o perjuicio de un principio, a él debe seguir en un segundo paso la comprobación de la importancia de la realización del principio contrario. En un tercer paso finalmente debe averiguarse si la importancia de la realización del principio contrario justifica el perjuicio o incumplimiento del otro.

Del análisis de la Figura 6: según la investigación realizada se ha obtenido que del $100 \%$ de los estudiantes encuestados un $80.6 \%$ considera que los programas de espectáculos y/o reality shows no contribuyeron a la realización de sus labores académicas, mientras el 19.4\% considera que sí.

Dicho resultado encuentra congruencia con el principio de "fomento de la educación, cultura y moral de la Nación" de la Ley de Radio y Televisión, Ley $\mathrm{N}^{\circ}$ 28278; el que es concordante con el articulo $\mathrm{N}^{\circ} 04$ de la misma ley: "Los servicios de radiodifusión tienen por finalidad satisfacer las necesidades de las personas en el campo de la información, el conocimiento, la cultura, la educación y el entretenimiento, en un marco de respeto de los deberes y derechos fundamentales, así como de promoción de los valores humanos y de la identidad nacional".

Además, se debe mencionar que la televisión es el medio de mayor alcance a nivel nacional, el que al mismo tiempo cuenta con la obligación de coadyuvar con el desarrollo de la sociedad, en el sentido de brindar una programación que enriquezca la cultura, e inspire el respeto por los valores de la sociedad.

Los argumentos presentados líneas arriba, encuentran coincidencia entre lo desarrollado ampliamente por el Tribunal Constitucional en diversas sentencias, siendo una de ellas, la sentencia $\mathrm{N}^{\circ}$ 2018-2015 AA, en la cual menciona que el derecho a la educación es un derecho fundamental, que dará paso al acceso a otros derechos fundamentales, así como también a la participación plena de la niñez y adolescencia en la vida política dentro de la sociedad, ante lo cual, la presente 
investigación determinó que en efecto se está vulnerando el derecho a la educación, ante la emisión de programas, que mitigan el desarrollo intelectual y analítico de los estudiantes de $1^{\circ}$ de secundaria de la I.E.E. "San Juan de la Libertad" de Chachapoyas.

Así mismo, la educación ostenta prelación del más alto rango, pues se fundamenta en los principios esenciales de la democracia y se vincula directamente con el desarrollo económico y social del país. Es también democrática porque se trata de un sistema de vida fundado en el constante mejoramiento económico, social y cultural del pueblo; está dirigida a la comprensión de nuestros problemas, al aprovechamiento de nuestros recursos, a la defensa de nuestra independencia política, al aseguramiento de nuestro progreso económico y a la continuidad y acrecentamiento de nuestra cultura, contribuyendo así a la mejor convivencia humana. Debe estar dirigida a fortalecer en la persona humana los principios de solidaridad, justicia social, la dignidad humana y la integridad de la familia. (Expediente $\mathrm{N}^{\circ}$ 04232-2004-AA. La educación y su rol en el desarrollo integral de la persona, Fundamento Jurídico $\mathrm{N}^{\circ} 10$, Tribunal Constitucional).En relación con la finalidad constitucional de la educación la Carta Fundamental señala expresamente que su propósito último debe ser "el desarrollo integral de la persona humana" (artículo 13). A partir de esta finalidad de desarrollo integral de la persona, la Constitución prevé distintas disposiciones dirigidas a darle contenido y a precisar los deberes estatales vinculados con ella. Señala, en este sentido, que a través de la educación se "promueve el conocimiento, el aprendizaje y la práctica de las humanidades, la ciencia, la técnica, las artes, la educación física y el deporte ", precisa que la educación "prepara para la vida y el trabajo y fomenta la solidaridad" (artículo 14). Dispone, asimismo, que forma parte de su contenido indisponible la "formación ética y cívica y la enseñanza de la Constitución y de los derechos humanos" (artículo 14), y que los educandos tienen derecho "a una formación que respete su identidad, así como al buen trato psicológico y físico" (artículo 15). En suma, se prevé una educación orientada al desarrollo integral de los educandos, el cual no se basa solo en saberes académicos, sino en la formación plena y multidimensional de las personas, en los diversos ámbitos de su vida personal y comunitaria, con base en el respeto a los derechos y los bienes constitucionales, formación integral que vincula de manera fuerte al Estado. Este Tribunal ha señalado, en este sentido, que el derecho a la educación "presupone un proceso de transmisión del saber y la afirmación de valores que ayuden a la persona en su desarrollo integral y en la realización de sus proyectos de vida en comunidad" (Expediente $\mathrm{N}^{\circ}$ 04232-2004-AA Naturaleza jurídica de la educación. La educación como derecho fundamental y como servicio público $\mathrm{N}^{\circ} 10$, Tribunal Constitucional).

En ese sentido la niñez y adolescencia forman parte del interés prioritario del Estado, quien dirige sus políticas, hacia la protección de la niñez y adolescencia: "cabe precisar que la protección especial brindada a niños, niñas y adolescentes no significa, de alguna forma, que les pueda considerar como un mero objeto de protección, sino más bien como auténticos sujetos de derechos. En este sentido, la protección que se les brinda no debe basarse únicamente en su situación de debilidad o vulnerabilidad, (...). Por el contrario, su protección parte de reconocerlas como personas y está encaminada a la construcción y al fortalecimiento progresivo de su autonomía, así como a la asunción de responsabilidades como futuro ciudadano (doctrina de la "protección integral"). De este modo, la protección especial a favor de los niños y adolescentes está encaminada a fortalecer y permitir el despliegue capacidades, así como a promover su bienestar, y jamás a su anulación o subordinación." (Expediente $\mathrm{N}^{\circ}$ 2018-2015-AA. Interés superior del niño, Fundamento jurídico $\mathrm{N}^{\circ} 20$, Tribunal Constitucional).

Lo anterior mencionado es reconocido por normas internacionales, tales como la Declaración Universal de los Derechos Humanos que en su Art. N 26 indica que la educación es un derecho que nos atañe a todos y es deber del Estado su protección y promoción, con el objeto del desarrollo pleno de la personalidad humana,

Desde otra óptica, en el mismo lineamiento, está el Pacto Internacional de Derechos Económicos, Sociales y Culturales; en su artículo 13, menciona que los Estados partes en el presente Pacto reconocen el derecho de toda persona a la educación; como segmento fundamental de su desarrollo en el proceso de ser ciudadano

En esa línea de ideas, la Convención sobre los Derechos del Niño en su artículo 28 menciona que los Estados partes reconocen el derecho del niño a la educación $\mathrm{y}$, a fin de que se pueda ejercer progresivamente y en condiciones de igualdad de oportunidades de ese derecho, es así como se genera una obligación imperativa al Estado peruano brindar una educación de calidad, que permita el progreso de nuestra generación y de generaciones futuras. 


\section{CONCLUSIONES}

5.1. Se determinó la existencia de vulneración al derecho a la educación por parte de los espectáculos y reality shows, en la televisión de señal abierta, además la transgresión al deber de colaboración de los medios de comunicación con el derecho fundamental a la educación, en concordancia con la Sentencia del Tribunal Constitucional $N^{\circ} 0091-$ 2005-AA, así como también al principio de protección y formación integral de los niños y adolescentes y el respeto de la institución familiar establecido en la Ley $\mathrm{N}^{\circ} 27287$, Ley de Radio y Televisión.

5.2. El tipo de entretenimiento que más sedujeron a observar a los estudiantes de $1^{\circ}$ de secundaria de la I.E.E. "San Juan de la Libertad", Chachapoyas, 2019, fueron los programas espectáculos y reality shows, transmitidos en televisión de señal abierta; lo que sin duda alguna contraviene la obligación que tiene del Estado, de proteger el derecho fundamental a la educación (en concordancia con la Sentencia del Tribunal Constitucional $\mathrm{N}^{\circ} 00853-2015$ ), ya que los programas espectáculos y reality shows son agentes vulnerantes, que merman la capacidad crítica y analítica de los estudiantes de la muestra estudiada.

5.3. Se demostró la influencia negativa de los programas de espectáculos y reality shows, emitidos en televisión de señal abierta, en el derecho a la educación en estudiantes del $1^{\circ}$ de secundaria de la I.E.E. "San Juan de la Libertad", Chachapoyas, 2019; hecho que vulnera el deber establecido en el artículo 14 de la Constitución Política del Perú de colaborar con el Estado en la educación y en la formación moral y cultural, además de contravenir los principio para la prestación de los servicios de radiodifusión, recogido en la Ley $\mathrm{N}^{\circ} 28278$ Ley de radio y Televisión ; en cuanto a que no se está fomentando la educación, cultura y moral de la nación.

5.4. Los espectáculos y reality shows vulneraron el horario de protección al menor establecido en el artículo 40 de la ley 28278, Ley de Radio y Televisión, demostrándose esa manera la inexistencia de políticas públicas destinadas a la regulación de los servicios de radiodifusión.

5.5. Se evidencio que el carácter ponderativo de derecho a la educación es mínimo respecto a la libertad de empresa, libertad de expresión y de opinión; en vista a que se ha demostrado que la emisión de programas de espectáculos y reality shows, es de transmisión continua, sin injerencia alguna del Estado orientada al respeto constitucional del derecho a la educación de la niñez y adolescencia como fin supremo del estado, permitiendo su transmisión sin una regulación eficaz, mientras que la televisión envuelve una millonaria empresa en su quehacer diario.

\section{REFERENCIAS BIBLIOGRÁFICA}

Alexy, R. (2010). Derechos fundamentales, ponderación y racionalidad. In El canon neoconstitucional (pp. 106-116). Trotta.

Centeno, R. (2016). "El caso de los programas juveniles Combate y Esto Es Guerra". (Tesis pre grado). Universidad Nacional Mayor de San Marcos. Lima-Perú.

Galvez, J. (2015). Preceptos normativos que respaldan al Estado peruano para regular el derecho a la libertad de expresión, permitiendo la erradicación de la televisión basura. Huancayo. (Tesis pre grado). Universidad Peruana los Andes. HuancayoPerú.

Gobierno Regional de Amzonas. (2007). Proyecto Educativo Regional de Amazonas 20072021. Chachapoyas.

Ley N ${ }^{\circ} 28278$ Ley de Radio y Televisión. (23 de junio de 2004). Diario oficial el Peruano. Lima, Perú.

Pintado, J. (2015). "Análisis del impacto de los contenidos discriminatorios y violentos en los programas de televisión y su incidencia en el comportamiento de los adolescentes del colegio San José la Salle, 2015". (Tesis pre grado). Universidad de Guayaquil. Guayaquil-Ecuador.

Sentencia del Tribunal Constitucional Expediente N. ${ }^{\circ}$ 0091-2005- AA. Determinación del contenido del derecho a la educación, Fundamento Jurídico $N^{\circ}$ 06, Tribunal Constitucional. Recuperado de https://tc.gob.pe/jurisprudencia/2006/0009 1-2005-AA.html

Sentencia del Tribunal Constitucional Expediente N. ${ }^{\circ}$ 2018-2015- AA. Interés superior del niño, Fundamento Jurídico $N^{\circ} 20$, Tribunal Constitucional. Recuperado de https://tc.gob.pe/jurisprudencia/2018/0201 8-2015-AA.pdf

Sentencia del Tribunal Constitucional Expediente N. ${ }^{\circ}$ 04232-2004- AA. La educación y su rol en el desarrollo integral de la persona, Fundamento Jurídico $N^{\circ} 10$, Tribunal Constitucional. Recuperado de http:/www.tc.gob.pe/jurisprudencia/2006/ 
04232-2004-AA.pdf

Sentencia del Tribunal Constitucional Expediente N. ${ }^{\circ}$ 00853-2015-AA. Las dimensiones de disponibilidad y accesibilidad del derecho a la educación como obligaciones estatales de respetar, proteger y cumplir, Fundamento Jurídico $N^{\circ} 37$, Tribunal Constitucional. Recuperado de https://tc.gob.pe/jurisprudencia/2017/0085 3-2015-AA.pdf

Tomaševski, K. (2000). Informe anual de la Relatora Especial sobre el derecho a la educación. Naciones Unidad. Comisión de Derechos Humanos.

Velázquez y Rey (2007). Metodología de la investigación científica: San Marcos E.I.R.L. Lima.

Villón, L. (2016). Los programas televisivos de reality de competencia (bln de canal uno y combate de canal rts) y su incidencia en el comportamiento de los y las adolescentes (entre 13 a 16 años de edad) en las unidades educativas fiscales (dr. Teodoro Alvarado Olea y veintiocho de mayo) del cantón Guayaquil. (Tesis pre grado). Universidad Laica Vicente Rocafuerte de Guayaquil. Guayaquil-Ecuador 